\title{
The De-Quervain Tenosynovitis: Literature Review
}

\author{
Bachar Chaya, Elsa Bakhach and Joseph Bakhach* \\ Division of Plastic \& Reconstructive Surgery, Hand \& Microsurgery, American University of Beirut Medical Center, Lebanon
}

Received: 眥 August 27, 2018; Published: 眥 September 05, 2018

*Corresponding author: Bakhach J, American University of Beirut Medical Center, P.O. Box 11-0236, Riad El-Solh, Beirut 1107-2020, Lebanon

\begin{abstract}
De Quervain tenosynovitis is a chronic, debilitating condition that is more common in females. Multiple modalities of treatment have been proposed and no definitive guidelines have been set yet. In this paper, we present the different treatment options for this condition. Non-surgical treatment consists mainly of anti-inflammatories, steroids and splinting. Surgically, different techniques have been described but with limited success. However, the authors of this paper have introduced a new technique the " $\Omega$ " Omegaplasty that is very promising in treating this chronic condition.
\end{abstract}

\section{Introduction}

All the extensor muscles of the forearm have a common origin known as the lateral epicondyle of the Humerus. In order to extend the wrist or the thumb, the dorsal (extensor) muscles of the forearm must contract. At the level of the wrist, the tendons of these muscles will separate into six different compartments. Each one of these compartments will be encased in a fibro-osseous sheath [1]. De Quervain tenosynovitis is a rheumatological condition caused by the entrapment of the first dorsal compartment of the wrist. This first compartment includes the tendon of both the Abductor Pollicis Longus (APL) and Extensor Pollicis Brevis (EPB) [1]. It was first described in 1895 by Fritz de Quervain as a "stenosing fibrous tendovaginitis" which is a non-inflammatory thickening of the tendons [1]. It is a common cause of wrist pain in adults and can become debilitating. Patients will usually complain of tenderness at the radial side of the wrist that is referred to the thumb or the lateral forearm [2].

The exact etiology of this condition is still under investigation. However, it has been attributed to either a repetitive microtraumas (caused by repetitive wrist and thumb extension) or to a major trauma to the APL and EPB tendons [3]. As a consequence, the synovial lining will undergo a myxomatous degeneration and fibrosis that will result in the thickening of the tendon sheath. This chronic thickening will eventually entrap the APL and EPB tendons producing pain and disability [4]. Eichoff maneuver and Finkelstein's test are diagnostic maneuvers of De Quervain tenosynovitis [5]. Both of them are performed while the patient is holding his thumb in his fist while the examiner deviates the hand to the ulnar side. If the patient's pain is reproduced, then De Quervain tenosynovitis is the most likely diagnosis [5].

\section{Epidemiology}

De Quervain tensosynovitis is relatively common condition that is more abundant in females with an incidence of $1.3 \%$ vs $0.5 \%$ in males [6]. It is much more prevalent in adults (Average age 50) and has no racial predilection [7]. New mothers report a higher incidence level then the rest of the population [8]. It is mainly due to the inappropriate way of lifting the newborns with the thumbs radially abducted and the wrist radially deviated [6]. Most of the cases will spontaneously resolve with conservative management, while some will require medical treatment or even surgery.

\section{Treatment}

The diagnosis of the tenosynovitis of the (APL) and (EPB) of the thumb, referred to as chronic stenotic tenosynovitis, is a mainly a clinical diagnosis [9]. In atypical presentation, an X-Ray of the wrist might be ordered in order to rule out other etiologies of wrist pain, like osteoarthritis of the joints of the thumb.

Treatment of this condition ranges from conservative treatment (splinting, analgesics...) to surgical. However, most of the cases will resolve spontaneously without the need of any treatment.

\section{Non-Surgical Management}

Non-surgical treatment should be our first modality of therapy. Anti-inflammatory, steroids, and splinting are the mainstay of 
treatment. Splinting was shown to be the least effective due to low level of compliance and the high rate of recurrence once the splint is removed. On the other hand, half of the patients will report improvement in the quality of the pain after the first corticosteroid injection and almost $90 \%$ will report improvement after the second injection [10]. For the remaining 10\%, surgical management is the only remaining option. The steroids should be injected into the fibro-osseous tunnel of the APL and EPB to prevent any side effects on the adjacent tissues (Subcutaneous tissue atrophy or hypo-pigmentation) [11]. A double blinded randomized controlled trial done by Chadderdon et.al demonstrated preliminary evidence about the superiority of bethamethasone injection over ketorolac injection in the treatment of De Quervain tenosynovitis. However, further studies are required for more definitive answers [12].

\section{Surgical Management}

Multiple techniques have been used to try and treat De Quervain tenosynovitis. The classical technique and the most widely used consists of dissecting longitudinally the apical level of the radial styloid pulley [13]. This will release the EPB tendon and expose the internal septum, liberating the heads of the APL tendon [14] However, this method is associated with multiple complications with the most commonly occurring being the subluxation of the EPB and APL tendons [15]. Multiple authors attempted to develop a new technique that prevents this subluxation but with limited success. The authors of this paper developed a new technique: The " $\Omega$ " Omegaplasty [16]. When looking at the first extensor compartment in a transverse section, you can notice that the pulley has particular " $\Omega$ " shape. The Omegaplasty procedures consists of expanding the internal volume of the pulley by releasing it from its anterior attachment to the anterior lip of the radial styloid. According to the case series done by Bakhach et.al on 25 patients with De Quervain tenosynovitis: "The described technique (Omegaplasty) is simple, reliable and respects the extensor tendons gliding physiology and biodynamics. By preserving the anatomical continuity of the first extensor compartment pulley at the wrist, the risk of adhesion formation is reduced." They have also noticed that the rate of the complication of subluxation, previously described, is much lower in their clinical series [16].

\section{Conclusion}

De Quervain tenosynovitis is chronic condition that when refractory to medical management is very difficult to treat. Multiple surgical techniques have been postulated. The author of this paper described a new technique: the Omegaplasty, that might become a potential surgical option for this debilitating condition.

\section{References}

1. Satteson E, Tannan SC (2018) De Quervain Tenosynovitis, in StatPearls. Treasure Island (FL).

2. Perno Ioanna D, Papaloizos M (2016) A comprehensive approach including a new enlargement technique to prevent complications after De Quervain tendinopathy surgery. J Hand Surg Rehabil 35(3): 183-189.

3. Stahl S, Vida D, Meisner C, Stahl AS, Schaller HE, et al. (2015) Work related etiology of de Quervain's tenosynovitis: a case-control study with prospectively collected data. BMC Musculo Skelet Disord 28(16): 126.

4. Huisstede BM, Gladdines S, Randsdorp MS, Koes BW (2018) Effectiveness of Conservative, Surgical, and Postsurgical Interventions for Trigger Finger, Dupuytren Disease, and De Quervain Disease: A Systematic Review. Arch Phys Med Rehabil 99(8): 1635-1649.

5. Goubau JF, Goubau L, Van Tongel A, Van Hoonacker P, Kerckhove D, et al. (2014) The wrist hyperflexion and abduction of the thumb (WHAT) test: a more specific and sensitive test to diagnose de Quervain tenosynovitis than the Eichhoff's Test. J Hand Surg Eur Vol 39(3): 286-292.

6. Stahl S, Vida D, Meisner C, Lotter O, Rothenberger J, et al. (2013) Systematic review and meta-analysis on the work-related cause of de Quervain tenosynovitis: a critical appraisal of its recognition as an occupational disease. Plast Reconstr Surg 132(6): 1479-1491.

7. Wolf JM, Sturdivant RX, Owens BD(2009) Incidence of de Quervain's tenosynovitis in a young, active population. J Hand Surg Am 34(1): 112115 .

8. Skoff HD (2001) "Postpartum/newborn" de Quervain's tenosynovitis of the wrist. Am J Orthop (Belle Mead NJ) 30(5): 428-430.

9. (2010) Tendon trouble in the hands: de Quervain's tenosynovitis and trigger finger. Women are more likely than men to develop these painful conditions. Harv Womens Health Watch 17(8): 4-5.

10. Oh JK, Messing S, Hyrien O, Hammert WC (2017) Effectiveness of Corticosteroid Injections for Treatment of de Quervain's Tenosynovitis. Hand (NY) 12(4): 357-361.

11. Khoo A, Grattan CE (2016) Making a dent with corticosteroid injections for de Quervain's tenosynovitis. BMJ Case Rep.

12. Chadderdon C, R Glenn Gaston, Bryan J Loeffler, Dan Lewis (2017) Betamethasone Versus Ketorolac Injection for the Treatment of De Quervain's Tenosynovitis: A Double-Blind Randomized Clinical Trial: Level 1 Evidence. J Hand Surg 42(9): S45-S46.

13. Bouras Y, El Andaloussi Y, Zaouari T, Touil N, Fnini S, et al. (2010) [Surgical treatment in De Quervain's tenosynovitis. About 20 cases]. Ann Chir Plast Esthet 55(1): 42-45.

14. Garcon JJ, Charruau B, Marteau E, Laulan J, Bacle G (2018) Results of surgical treatment of De Quervain's tenosynovitis: 80 cases with a mean follow-up of 9.5 years. Orthop Traumatol Surg Res.

15. Bering J, Bignion D, Kurzen P (2013) [Enlargement plasty of the first dorsal compartment for the surgical treatment of de Quervain's tenosynovitis]. Oper Orthop Traumatol 25(4):361-370.

16. Bakhach J, Chaya B, Papazian N (2018) Omega Pulley Plasty for Surgical Management of DeQuervain's Disease. J Hand Surg Asian Pac Vol 23(2): 170-175. 
ISSN: 2574-1241

DOI: 10.26717/BJSTR.2018.08.001691

Pablo Camacho Lazarraga. Biomed J Sci \& Tech Res

(c) (P) This work is licensed under Creative

Submission Link: https://biomedres.us/submit-manuscript.php

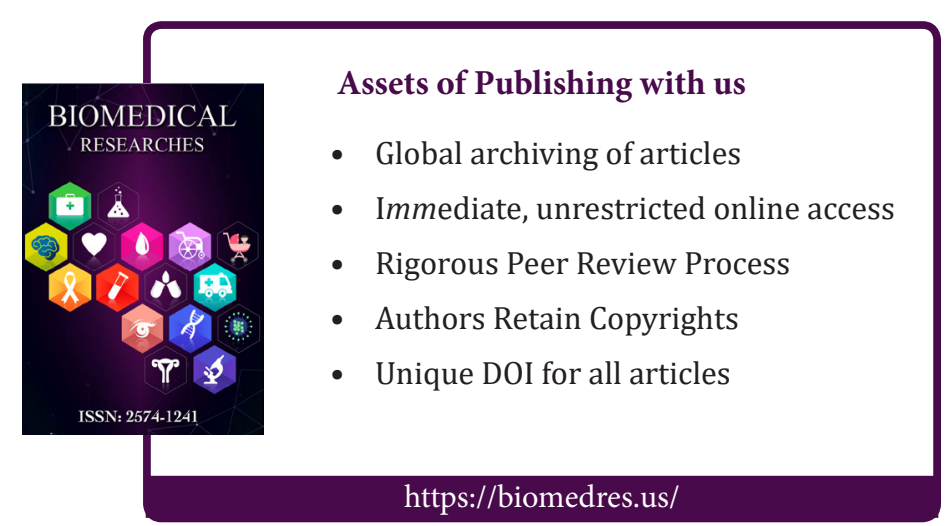

